\title{
RACK1 downregulation suppresses migration and proliferation of neuroblastoma cell lines
}

\author{
FAN LU, CHI ZHANG, WEN-JIE WU and YE-MING WU \\ Department of Pediatric Surgery, Shanghai Xinhua Hospital, Affiliated with \\ Shanghai Jiao Tong University, Shanghai 200092, P.R. China
}

Received October 26, 2011; Accepted December 22, 2011

DOI: 10.3892/or.2012.1629

\begin{abstract}
Neuroblastoma is the most common extracranial solid tumor in childhood, whose molecular mechanism on clinically heterogeneous behavior is still unclear. Receptor for activated C-kinase 1 (RACK1) has been shown to be involved in the regulation of growth and migration in many types of cells. Two types of human neuroblastoma cell lines, SK-N-SH and SK-N-BE (2), were used to explore the role of RACK1 in neuroblastoma cell migration and proliferation. Cell migration and proliferation were detected by a transwell system and colorimetric bromodeoxyuridine (BrdU) ELISA, respectively. The expressions of RACK1, N-Myc, phospho-Src(Tyr416), phospho-Src(Tyr527), phospho-Akt, phospho-ERK1/2 and phospho-p38 were analyzed by Western blotting. The expression of RACK 1 was then repressed by target RNA interference (RNAi) in both types of neuroblastoma cells, and the expression of signaling molecules, migration and proliferation of cells was analyzed. Compared with SK-N-SH, the migration and proliferation of SK-N-BE(2) cells was remarkably higher which was accompanied by higher expression of RACK1 and phospho-Src(Tyr416). RACK1 RNAi repressed cell migration and proliferation, and decreased the expression of phosphoSrc(Tyr416) in both cell lines. In summary, RACK1 was expressed in the neuroblastoma cells and positively regulated cell migration and proliferation probably via modulating the activation of Src on Tyr416 residue.
\end{abstract}

\section{Introduction}

Neuroblastoma, a neoplasm of peripheral neural crest origin, is the most common extracranial solid tumor in childhood and the most frequently diagnosed neoplasm during infancy $(1,2)$. Despite current therapeutic advances and basic mechanism

Correspondence to: Dr Ye-Ming Wu, Department of Pediatric Surgery, Xinhua Hospital, Affiliated with Shanghai Jiao Tong University, 1665, Kong Jiang Road, Yangpu, Shanghai 200092, P.R. China

E-mail: doctorl2005@yahoo.com.cn

Key words: receptors for activated C-kinases, neuroblastoma, migration, proliferation investigations, neuroblastoma remains a complex medical challenge with the unpredictable clinical course and dismal overall outcome for advanced-stage disease (3-5).

Extraordinary migration and proliferation plays an important role in invasive and malignant of neuroblastoma cells $(6,7)$. It is proposed that finding key point proteins or signaling molecules that participated in the invasive potential, such as migration and proliferation, of neuroblastoma cells, might be a potential therapeutic targets and might be a way to overcome current clinical dilemma of neuroblastoma treatments (8).

Receptors for activated C-kinases (RACK1) is the founding member of the family of intracellular receptors of activated protein kinase C (PKC) $(9,10)$. The human RACK1 gene (Gene Bank accession no. GNB2L1) is highly conserved, includes eight exons and seven introns and is located on chromosome 5 (5q35.3). The gene product is a $36-\mathrm{kDa}$ cytosolic protein that contains seven tryptophan-aspartate repeats, a domain involved in protein-protein interactions. RACK1 is widely expressed in the nervous system (11). Several studies suggested that RACK1 are involved in numerous key cellular functions, such as cellular apoptosis (9) and migration (12). It had been reported that the expressions of RACK1 mRNA and protein were upregulated in damaging and repairing process (13) and during angiogenesis in carcinomas (14). In light of the role in cell growth, apoptosis and proliferation, there are accumulating interest focused on the oncogenic role of RACK1 signaling (15-19). Hence, we hypothesized that RACK1 might play an important role in the development and progression of the neuroblastoma. However, few works were carried out to evaluate the expression and function of the RACK1 in the growth and migration of neuroblastoma.

In the present research, the expression of RACK1, and the migration and proliferation in two kinds of human neuroblastoma cell lines, SK-N-SH and SK-N-BE(2), were detected. The possible role of RACK1 in migration and proliferation of neuroblastoma cells were further analyzed after RACK1 gene silence. Our results could provide a useful tool for the evaluation of the invasive potential of neuroblastoma and may provide a new therapeutic target.

\section{Materials and methods}

Cell culture. The SK-N-SH and SK-N-BE(2) cell lines were purchased from cell bank of Chinese Academy of 
Science. SK-N-SH is a MYCN gene (gene name of N-Myc) non-amplified cell line while SK-N-BE(2) is a MYCN gene amplified cell line (20). SK-N-SH was cultured in Dulbecco's modified Eagle's medium (DMEM, Invitrogen) containing $10 \%$ fetal bovine serum (FBS, Invitrogen), $100 \mathrm{U} / \mathrm{ml}$ penicillin and $100 \mu \mathrm{g} / \mathrm{ml}$ streptomycin and SK-N-BE(2) was cultured in advanced DMEM/Nutrient Mixture F-12 (DMEM/F12, Invitrogen) containing 10\% FBS (Invitrogen), $100 \mathrm{U} / \mathrm{ml}$ penicillin and $100 \mu \mathrm{g} / \mathrm{ml}$ streptomycin. Cells were incubated at $37^{\circ} \mathrm{C}$ in a humidified incubator of $95 \%$ air and $5 \% \mathrm{CO}_{2}$.

RNA interference. The mRNA sequences of human RACK1 [NM_006098, Homo sapiens guanine nucleotide binding protein (G protein), $\beta$ polypeptide 2-like 1 (GNB2L1)] and N-Myc [NM_005378, Homo sapiens v-myc myelocytomatosis viral related oncogene, neuroblastoma derived (avian) (MYCN)] were acquired from NCBI GenBank. Small interfering RNAs (siRNA) target against human RACK1 and N-Myc mRNA were designed and synthesized by GenePharma Biological Co. (Shanghai, China). The double strands of siRNA for RACK1 were: 5'-CCCACUUUGUUAGCGAUGUTT-3' and 5'-ACA UCGCUAACAAAGUGGGTT-3'. siRNA for N-Myc were: 5'-CGGACGAAGAUGACUUCUATT-3' and 5'-UAGAA GUCAUCUUCGUCCGTT-3'. For RNA interference (RNAi) studies, SK-N-SH and SK-N-BE(2) were seeded at a density of $2.0 \times 10^{5}$ cells per well in 6-well plate and grown in 10\% FBS/ DMEM and 10\% FBS/DMEM/F12, respectively. After seeding for $24 \mathrm{~h}$, the cells were transfected with siRNA and Lipofectamine $^{\mathrm{TM}} 2000$ (Invitrogen) according to the manufacturer's instruction. Briefly, $100 \mathrm{nmol}$ siRNA and $5 \mu \mathrm{l}$ Lipofectamine $^{\mathrm{TM}} 2000$ were diluted in opti-MEM (Invitrogen), serum- and antibiotic-free, to a final volume of $800 \mu$ l. After mixing for $20 \mathrm{~min}$ at room temperature, the siRNA/ Lipofectamine 2000 mixture was added dropwise onto the cells and incubated for $6 \mathrm{~h}$ at $37^{\circ} \mathrm{C}$ in a humidified $\mathrm{CO}_{2}$ incubator. Following incubation, the mixture was replaced with complete medium [10\% FBS/DMEM for SK-N-SH and $10 \%$ FBS/DMEM/F12 for SK-N-BE(2)] for $24 \mathrm{~h}$. Non-silencing siRNA that does not recognize any known homology to human genes was used as a mock control.

Real-time PCR and Western blotting. The mRNA expressions of RACK1 in different human neuroblastoma cell lines were detected by real-time PCR (Bio-Rad). Specific primers for N-Myc were synthesized by Sangon Biological Co. (Shanghai, China) (sense: 5'-GCTGGGTCACGGAGATGCT-3'; antisense: 5'-CGCTTGTTCACGGGAAAGG-3'; product length $373 \mathrm{bp})$. Specific primers for RACK1 were, sense: 5'-GAGCAA ATGACCCTTCGT-3'; antisense: 5'-GTAGTGCCCGTTGTG AGA-3'; product length $284 \mathrm{bp}$. Amplification was performed in parallel samples using human glyceraldehyde-3-phosphate dehydrogenase (GAPDH) primers (sense: 5'-GGCAGCCCA GAACATCATCC-3'; antisense: 5'-GCCAGCCCAAGCATC AAAG-3'; product length 298 bp) as a control.

For the Western blot analysis, cells were lysed by IP Lysis buffer (Themo) with protease inhibitor cocktail tablet (Roche Diagnostics) and phosphatase inhibitor cocktail tablet (Roche Diagnostics) freshly added. The protein concentrations were determined by the Bradford method (Bio-Rad). Then, proteins, $30 \mu \mathrm{g}$ per lane, were subjected to electrophoretic separation with $12 \%$ SDS-PAGE and transferred to nitrocellulose membranes (Hybond, Amersham). Western blots were performed by using antibodies directed against N-Myc (1:500, Cell Signaling Technologies), RACK1 (1:500, Abcam), GAPDH (1:500, Santa Cruz Biotechnology), phospho-Src(Tyr416) (1:500, Cell Signaling Technology), phospho-Src(Tyr527) (1:500, Cell Signaling Technology), total-Src (1:500, Cell Signaling Technology), phospho-Akt (1:500, Cell Signaling Technology), total-Akt (1:500, Cell Signaling Technology), phospho-p38 (1:500, Cell Signaling Technology), total-p38 (1:500, Santa Cruz Biotechnology), phospho-ERK1/2 (1:500, Cell Signaling Technology) and total-ERK1/2 (1:500, Cell Signaling Technology), respectively. After incubation with alkaline phosphatase conjugated secondary antibodies (Jackson Immunoresearch), the signals were visualized by nitroblue tetrazolium-bromochloroindolyl phosphate (Bio Basic, Inc.) and quantified by Quantity One software (Bio-Rad).

Cell migration assay. Cell migration assay was performed with the Transwell (Costar) system, which allows cells to migrate through a polycarbonate membrane with a pore size of $8 \mu \mathrm{m}$ in 6-well plates. Briefly, cells were plated at a concentration of $1.0 \times 10^{6}$ cells $/ \mathrm{ml}$ on the upper chamber and the lower chamber was filled with $800 \mu 120 \%$ FBS/DMEM for SK-N-SH and 20\% FBS/DMEM/F12 for SK-N-BE(2) to serve as the chemoattractant. After $6 \mathrm{~h}$ of incubation at $37^{\circ} \mathrm{C}$ in a humidified $\mathrm{CO}_{2}$ incubator, cells were fixed in $4 \%$ paraformaldehyde for $20 \mathrm{~min}$, permeabilized for 5 min with $0.4 \%$ Triton X-100 in PBS and dyed with Ehrlich's haematoxylin solution for $30 \mathrm{~min}$. The number of migrated cells was counted under a microscope (magnification x200, Olympus). Six randomly chosen fields were evaluated per transwell membrane.

Cell proliferation assay. Cell proliferation was analyzed using a colorimetric bromodeoxyuridine (BrdU) kit (Roche Diagnostics). SK-N-SH and SK-N-BE(2) were seeded at a density of $1.0 \times 10^{4}$ cells per well in 96 -well plates and grown in 10\% FBS/DMEM and 10\% FBS/DMEM/F12, respectively. $6 \mathrm{~h}$ prior to the end of RNAi, BrdU labeling reagent was added into the culture medium (1:1000) and labeled according to the manufacturer's instructions. Briefly, the cells were fixed with FixDenat solution for $30 \mathrm{~min}$ at room temperature and then incubated with anti-BrdU peroxidase working solution (freshly diluted 1:100) for $90 \mathrm{~min}$. Following 3 rinses with washing buffer, $100 \mu \mathrm{l} /$ well substrate solution was added onto the cells and incubated for $20 \mathrm{~min}$ at room temperature. Thereafter, $25 \mu 1 \mathrm{H}_{2} \mathrm{SO}_{4}, 1 \mathrm{~mol} / \mathrm{l}$, were added to each well and the 96-well plates were shaken at 300 r.p.m. The absorbance at $450 \mathrm{~nm}$ was measured in an ELISA plate reader (Bio-Rad 680).

Statistical analysis. Each experiment was performed at least in triplicates and all values are expressed as mean $\pm \mathrm{SD}$. The one-way ANOVA was used to compare the results between the two groups. A value of $\mathrm{P}<0.05$ was considered significant.

\section{Results}

High migration and proliferation properties of $S K-N-B E(2)$ with high expression of RACK1. The mRNA and protein expres- 

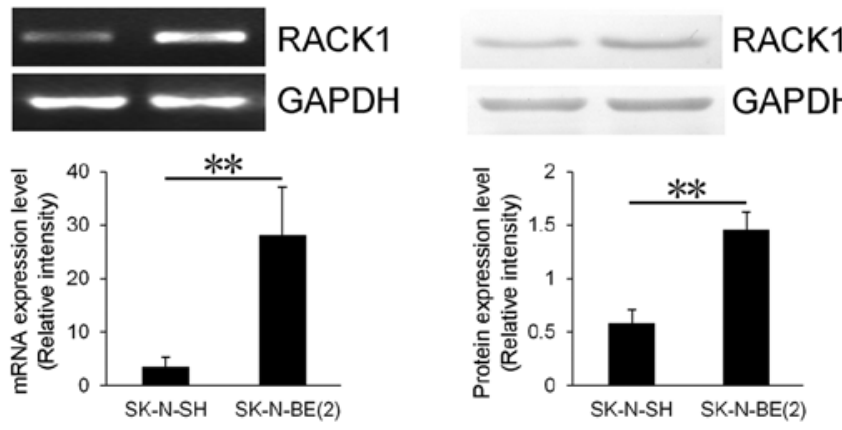

Figure 1. The mRNA and protein expressions of RACK1 were both significantly higher in SK-N-BE(2) than that in SK-N-SH. The top panel shows the gels (RT-PCR) and the blots (Western blotting) from a typical study; the bottom histogram shows the relative intensities of RACK1 which were densitometric quantified by relating to GAPDH. Values shown are the mean \pm SD for each condition from 4 independent experiments. ${ }^{* *} \mathrm{P}<0.01$ vs. SK-N-SH.

sions of RACK1 in SK-N-BE(2), the tumorigenic, aggressive and MYCN gene amplified neuroblastoma cell line, were both significantly higher than that in SK-N-SH, the less aggressive and MYCN gene non-amplified ( $\mathrm{P}<0.01$, Fig. 1). The migration property of SK-N-BE(2), analyzed by Transwell system, was notably higher than that of SK-N-SH (P<0.05, Fig. 2A). Cell proliferation was evaluated by measuring BrdU incorporation in newly synthesized DNA. As shown in Fig. 2B, in comparison with SK-N-SH, the proliferation of SK-N-BE(2) was also notably higher $(\mathrm{P}<0.05)$. These data suggested that RACK1 might play a role in neuroblastoma growth and migration.

Expressions of N-Myc, phospho-Src, phospho-Akt, phosphoERK1/2 and phospho-p38 in SK-N-SH and SK-N-BE(2). To evaluate the possible molecules involved in differential migration and proliferation of SK-N-BE(2) and SK-N-SH, the expression of N-Myc and the activations of Src, Akt, ERK1/2 and $\mathrm{p} 38$ were assessed by their specific antibody and phosphoantibodies individually. The phospho-antibodies recognize the phosphorylated Tyr416 and Tyr527 residue on Src, Thr308 residue on Akt, Thr180 and Tyr182 residues on p38, Thr202 and Tyr204 residues on ERK1/2. These phosphorylated residues are the critical sites required for the activation of these factors, respectively.

As shown in Fig. 3, the expression of N-Myc was significantly higher in MYCN amplified SK-N-BE(2) than that in MYCN non-amplified SK-N-SH $(\mathrm{P}<0.01)$. The phosphorylation of Src on Tyr416 residue was also increased in SK-N-BE(2) ( $\mathrm{P}<0.01$, Fig. 3), while on Tyr527 residue the phosphorylation was similar between the two cell lines ( $\mathrm{P}>0.05$, Fig. 3$)$. The phosphorylation on Akt was remarkably decreased in SK-N$\mathrm{BE}(2)$ in comparison with SK-N-SH $(\mathrm{P}<0.01$, Fig. 3). The expressions of phospho-ERK1/2 and phospho-p38 in SK-N$\mathrm{BE}(2)$ had no significant difference from that in SK-N-SH (P>0.05, Fig. 3).

The protein expressions of RACK1, N-Myc, phospho$\operatorname{Src}(\mathrm{Ty} r 416)$ were changed similar to, while phospho-Akt was opposite to, the migration and proliferation of cells. Therefore, it provided evidence that these factors might participate in the
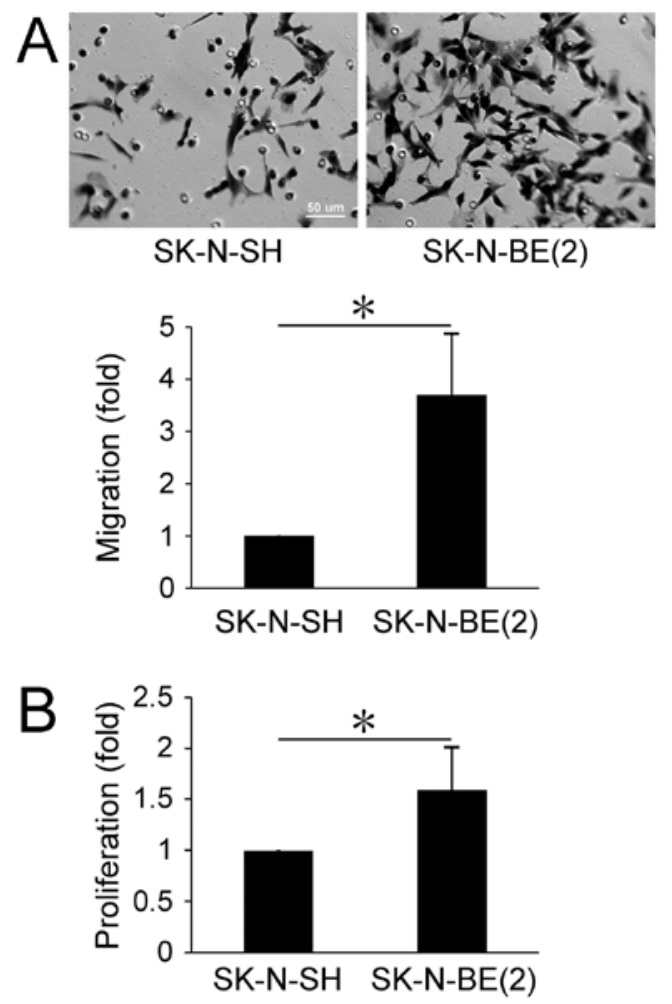

Figure 2. The migration and proliferation of SK-N-SH and SK-N-BE(2). (A) Compared with SK-N-SH, SK-N-BE(2) had a higher ability on migration, which was determined by Transwell assays. The number of cells illustrates the migration ability. Bar, $50 \mu \mathrm{m}$. Histogram shows fold changes in cell migration relative to $\mathrm{SK}-\mathrm{N}-\mathrm{SH}$. Values shown are the mean $\pm \mathrm{SD}$ for each condition from 3 independent experiments and 6 images were random acquired in each independent experiment. ${ }^{*} \mathrm{P}<0.05$ vs. SK-N-SH. (B) Compared with SK-N-SH, SK-N-BE(2) had a higher ability on proliferation, which was determined by BrdU ELISA. Histogram shows fold changes in cell proliferation relative to SK-N-SH. Values shown are the mean \pm SD for each condition from 4 independent experiments. ${ }^{*} \mathrm{P}<0.05$ vs. SK-N-SH.

modulation of migration and proliferation of neuroblastoma cells, but phospho-ERK1/2 and phospho-p38 might not.

Repressed expression of RACK1 on $S K-N-B E(2)$ downregulates the migration and proliferation of cell and decreases the phosphorylation of Src(Tyr416). In comparison with the mock control, the decreased expression of RACK1 (P<0.01, Fig. 4) by target RNAi significantly repressed migration and proliferation of SK-N-BE(2) (P<0.05, Fig. 5). Specific RACK1 RNAi also significantly suppressed the phosphorylation of Src on Tye416 residue $(\mathrm{P}<0.01$, Fig. 4$)$, but had no specific effects on the expression of N-Myc and phosphorylation of Src on Tye 527 residue, Akt, ERK1/2 and p38 (P>0.05, Fig. 4).

Repressed expression of RACK1 on SK-N-SH decreases the proliferation of cells and the phosphorylation of $\operatorname{Src}$ (Tyr416). In SK-N-SH, RACK1 'knock-down' by RNAi $(\mathrm{P}<0.01$, Fig. 6) notably repressed the phosphorylation of Src on Tye416 residue $(\mathrm{P}<0.05$, Fig. 6$)$, while the expression of phosphorylation of Src on Tye527 residue, Akt, ERK1/2 and p38 were also similar between the RACK1 RNAi and the mock control ( $P>0.05$, Fig. 6). Target RACK1 RNAi also decreased the cell proliferation $(\mathrm{P}<0.05$, Fig. $7 \mathrm{~B})$ and 


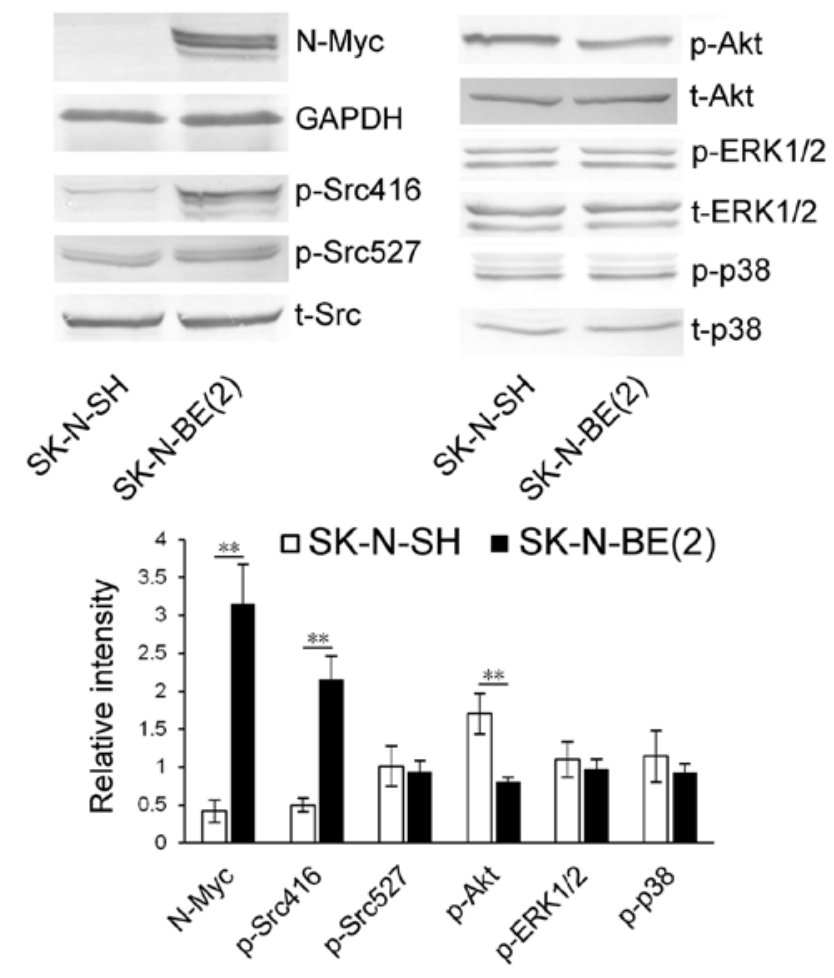

Figure 3. Expressions of N-Myc, and phosphorylations of Src(Tye416), Src(Tye527), Akt, ERK1/2 and p38 in SK-N-SH and SK-N-BE(2). The expression of N-Myc was significantly higher in SK-N-BE(2) than that in SK-N-SH, accompanied by remarkably increased expression of phospho-Src(Tye416) and decreased expression of phosphor-Akt. Top panel, the blots from a typical study; the bottom histogram shows the relative intensities of N-Myc which were densitometrically quantified by relating to GAPDH, and the relative intensities of phospho-Src(Tye416), phospho-Src(Tye527), phospho-Akt, phospho-ERK1/2 and phospho-p38, were densitometrically quantified by relating to their individual total proteins, respectively. Values shown are the mean \pm SD for each condition from 4 independent experiments. " $\mathrm{P}<0.05$ vs. SK-N-SH, ${ }^{* *} \mathrm{P}<0.01$ vs. SK-N-SH.

migration, but the decreased effect on cell migration was not significant ( $\mathrm{P}>0.05$, Fig. $7 \mathrm{~A})$.

Repressed expression of $N-M y c$ downregulates the migration and proliferation of $S K-N-B E(2)$, decreases expressions of RACK1, phosphor-Src(Tyr416), but increases expression of phospho-Akt. The role of N-Myc in the regulation of neuroblastoma cell proliferation and migration has been confirmed by several studies $(21,22)$. We then using RNAi techniques to suppress the expression of N-Myc in SK-N-BE(2) in order to detect the relation between N-Myc expression and RACK1 expression.

When the expression of N-Myc was remarkably suppressed by RNAi in comparison with the mock control ( $\mathrm{P}<0.01$, Fig. 4), the migration and proliferation of SK-N$\mathrm{BE}(2)$ were significantly decreased $(\mathrm{P}<0.05$, Fig. 5$)$. The expressions of RACK1 and phospho-Src(Tye416) were also decreased by N-Myc RNAi ( $\mathrm{P}<0.05$, Fig. 4), while expression of phospho-Akt was increased $(\mathrm{P}<0.05$, Fig. 4). The expressions of phospho-Src(Tye527), phospho-ERK1/2 and phospho-p38 were not changed by N-Myc RNAi in SK-N$\mathrm{BE}(2)$ ( $\mathrm{P}>0.05$, Fig. 4).
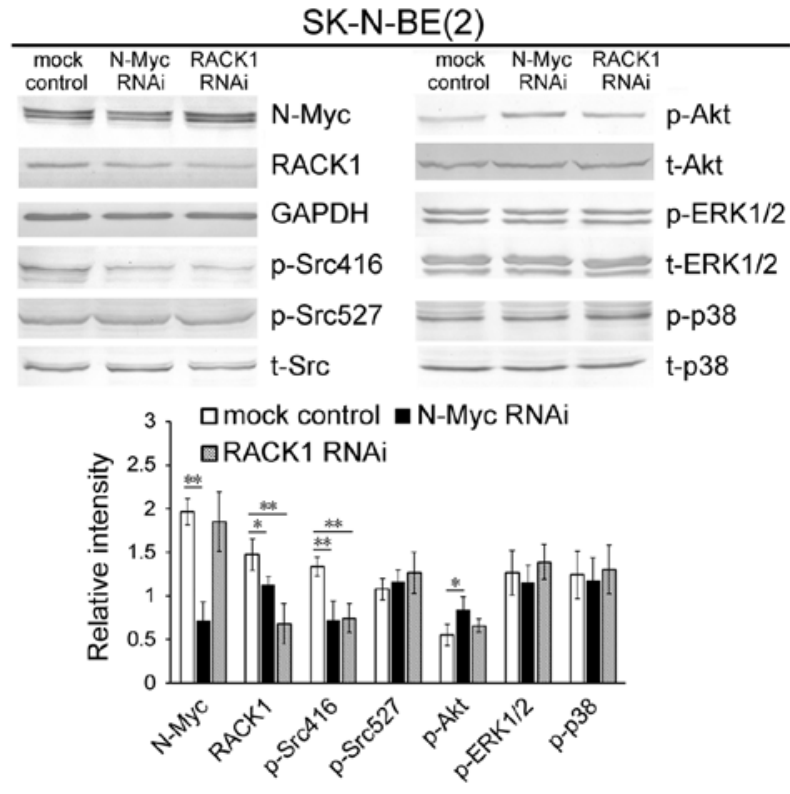

Figure 4. The effects of N-Myc and RACK1 target RNAi on expressions of N-Myc and RACK1, and phosphorylation of Src(Tye416), Src(Tye527), Akt, ERK1/2 and p38 in SK-N-BE(2). The expression of RACK1 was decreased by target N-Myc RNAi, but the expression of N-Myc was not changed by target RACK1 RNAi. The phosphorylation of Src Tye416 residues were decreased by both N-Myc and RACK1 RNAi, while the phosphorylation of Akt was only induced by N-Myc RNAi. The phosphorylation of Src Tye527 residues, ERK1/2 and p38 were not changed by N-Myc nor RACK1 RNAi. Values shown are the mean $\pm \mathrm{SD}$ for each condition from 4 independent experiments. ${ }^{*} \mathrm{P}<0.05$ vs. the mock control, ${ }^{* *} \mathrm{P}<0.01$ vs. the mock control.

\section{SK-N-BE(2)}

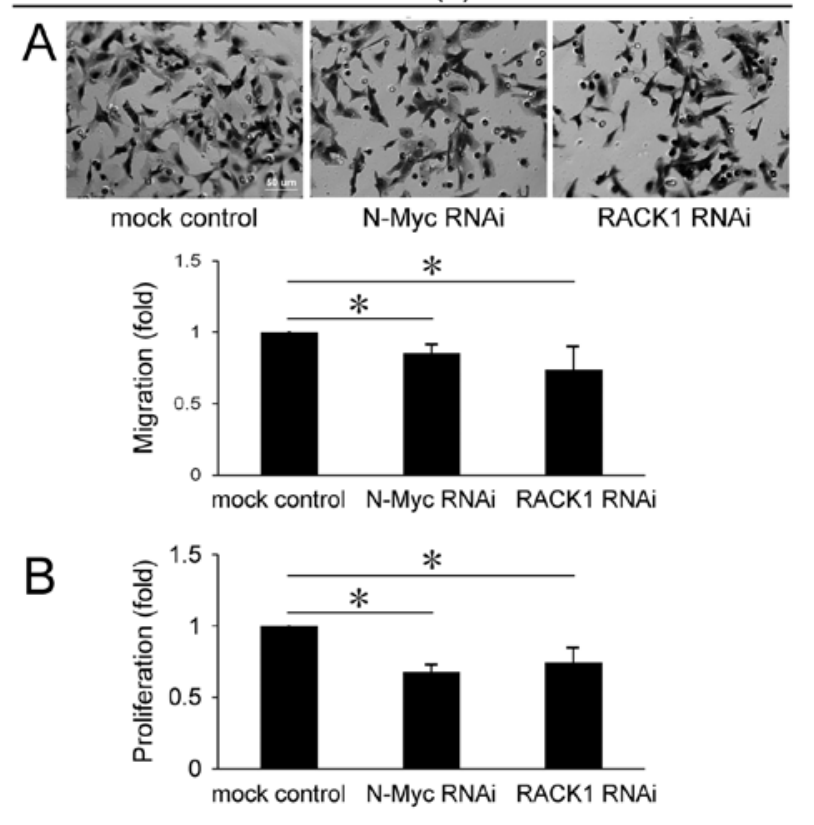

Figure 5. The effect of N-Myc and RACK1 target RNAi on migration and proliferation of SK-N-BE(2). (A) Compared with the mock control, both N-Myc and RACK1 target RNAi repressed the migration ability of SK-N-BE(2), which was determined by Transwell assays. The number of cells illustrates the migration ability. Bar, $50 \mu \mathrm{m}$. Histogram shows fold changes in cell migration relative to the mock control. Values shown are the mean \pm SD for each condition from 3 independent experiments and 6 images were random acquired in each independent experiment. ${ }^{*} \mathrm{P}<0.05$ vs. the mock control. (B) Both N-Myc and RACK1 target RNAi decreased the proliferation ability of SK-N-BE(2), which was determined by BrdU ELISA. Histogram shows fold changes in cell proliferation relative to the mock control. Values shown are the mean \pm SD for each condition from 4 independent experiments. ${ }^{*} \mathrm{P}<0.05$ vs. the mock control. 

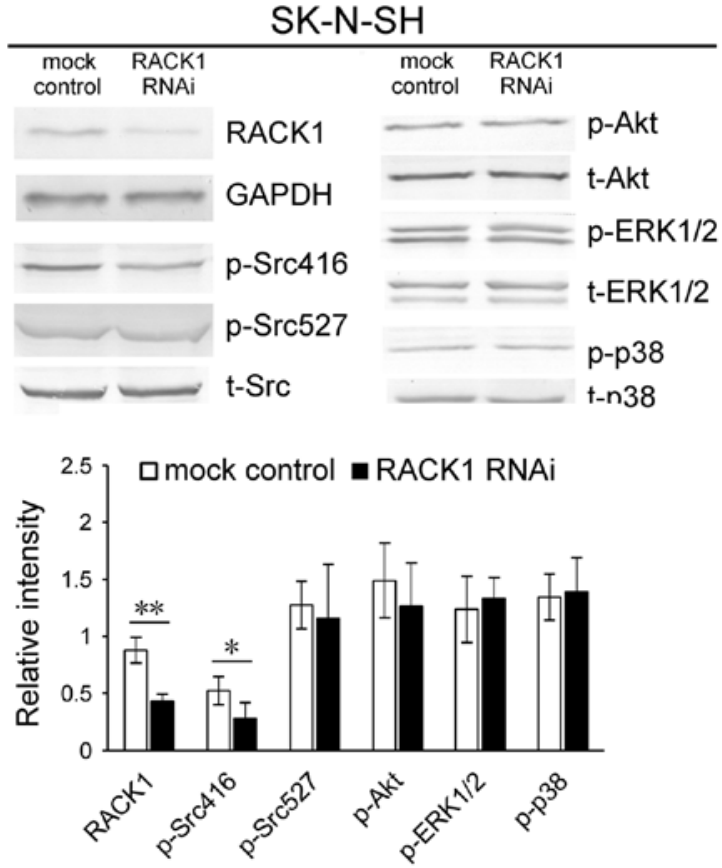

Figure 6. The effects of RACK1 target RNAi on expression of RACK1, and phosphorylation of Src(Tye416), Src(Tye527), Akt, ERK1/2 and p38 in SK-N-SH. Similar to the variation of signaling molecules in SK-N-BE(2), the expression of RACK1 was decreased by target RNAi. The phosphorylation of Src Tye416 residues were decreased by RACK1 RNAi. The phosphorylation of Src Tye527 residues, Akt, ERK1/2 and p38 were not changed by RACK1 RNAi. Values shown are the mean \pm SD for each condition from 4 independent experiments. ${ }^{*} \mathrm{P}<0.05$ vs. the mock control, ${ }^{* *} \mathrm{P}<0.01$ vs. the mock control.

The results revealed that the high expression of $\mathrm{N}-\mathrm{Myc}$ in SK-N-BE(2) was a crucial inducer for cell migration and proliferation. The expression of RACK1 and phosphorylation of $\operatorname{Src}($ Tye416) and Akt might be involved in the signaling mechanism of N-Myc induced migration and proliferation, but phosphorylation of Src(Tye527), ERK1/2 and p38 might not be involved in this process.

The expression of RACK1 was affected by N-Myc expression, but RACK1 had no effect on the expression of N-Myc. The present data suggest that RACK1 'knock-down' and N-Myc 'knock-down' had similar effects on SK-N-BE(2) migration, proliferation and Src(Tye416) phosphorylation, but different on Akt phosphorylation. Hence, RACK1 might be a downstream signaling molecule of N-Myc, and contributed to the migration and proliferation of SK-N-BE(2) induced by N-Myc. The phosphorylation of Src on Tye416 residue might be involved in this process, but Akt might not.

\section{Discussion}

Hyper-proliferation and hyper-migration behaviors induced by various oncogenic proteins are the most prominent and well-characterized features of neuroblastoma cells, and are fundamental to tumor growth and metastasis $(6,7)$. Finding new regulating mechanism might provide a useful tool for the evaluation of the invasive potential of neuroblastoma and might provide a new therapeutic target.

In this study, our results revealed that RACK1 was expressed in neroblastoma and was involved in regulation of

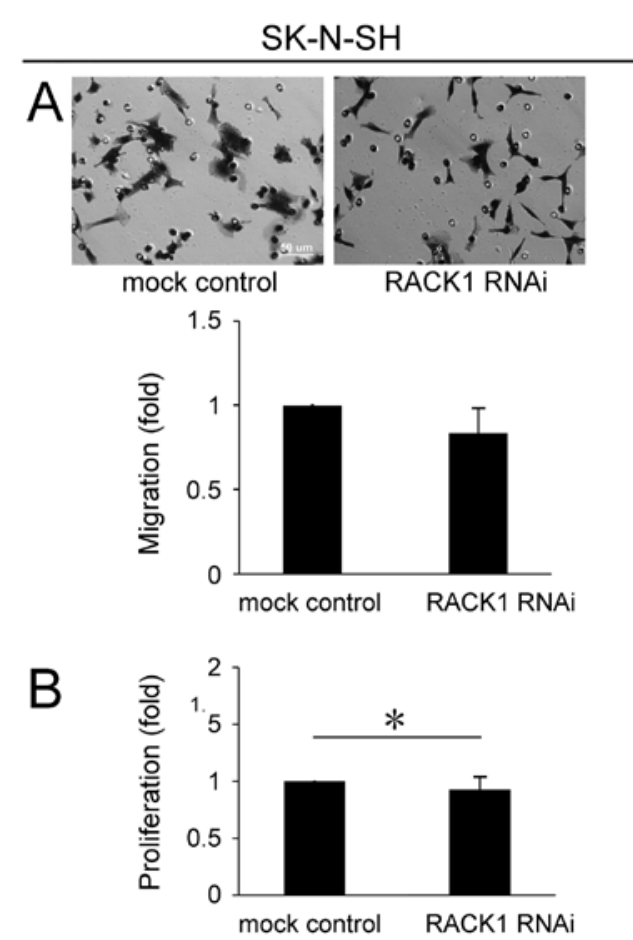

Figure 7. The effect of RACK1 target RNAi on migration and proliferation of SK-N-SH. (A) Compared with the mock control, RACK1 RNAi had no significant effect on cell migration, which was determined by Transwell assays. The number of cells illustrates the migration ability. Bar, $50 \mu \mathrm{m}$. Histogram shows fold changes in cell migration relative to the mock control. Values shown are the mean \pm SD for each condition from 3 independent experiments and 6 images were random acquired in each independent experiment. ${ }^{*} \mathrm{P}<0.05$ vs. the mock control. (B) Compared with the mock control, RACK1 target RNAi decreased the proliferation ability of SK-N-SH, which was determined by BrdU ELISA. Histogram shows fold changes in cell proliferation relative to the mock control. Values shown are the mean \pm SD for each condition from 4 independent experiments. " $\mathrm{P}<0.05$ vs. the mock control.

tumor cell migration and proliferation. Differential expression of RACK1 in the human neuroblastoma cell lines, SK-N-SH and SK-N-BE(2), were accompanied with differential abilities of migration and proliferation. The expression of RACK1, cell migration and proliferation were significantly higher in SK-N$\mathrm{BE}(2)$, an aggressive and MYCN gene (gene name of N-Myc) amplified neuroblastoma cell line, than that in SK-N-SH, a less aggressive and MYCN non-amplified neuroblastoma cell line. The RACK1 gene silencing by RNAi reduced cell proliferation in both cell lines and cell migration in SK-N-BE(2) cells significantly. The decreased effects of RACK1 gene silencing on migration of SK-N-SH was not significant, which might be due to the basic migration rate of SK-N-SH that was lower than SK-N-BE(2) and the Transwell assay for migration was less sensitive than the ELISA for proliferation. These data suggested that RACK1 could be a key regulator at least on neuroblastoma cell proliferation.

To evaluate the signaling molecules that may be involved in RACK1 induced migration and proliferation of neuroblastoma cell, the phosphorylation of Src, which interacts with RACK1 via binding with Src homology (SH2) domains $(11,12)$ and the phosphorylation of Akt, ERK1/2 and p38, which are involved in regulation of neuroblastoma migration, apoptosis and proliferation $(23,24)$ were detected. 
Our results revealed that the phosphorylation of Tyr416 residue on Src, but not Tyr527 residue, was regulated by RACK1 RNAi in both SK-N-SH and SK-N-BE(2). Since, Tyr416 for kinase active and Tyr527 for kinase inactive (25), repressed expression of RACK1 resulted in a decreased activation of Src kinase via decreased phosphorylation on Tyr416 residue of Src.

$\mathrm{Src}$ is a non-receptor protein tyrosine kinase, whose activity and expression has been strongly implicated in the development, maintenance, progression, and metastatic spread of several human cancers (26). Overexpression or aberrant activation of Src has been detected in neuroblastoma (27). Downregulation of Src activity was associated with apoptosis of neuroblastoma cells (28). There are reports that RACK1 negatively regulated cell growth and migration via Src-dependent pathways $(9,12)$.

MYCN gene has been proven to be a critical gene for stimulation of neuroblastoma growth and migration $(21,22)$. Our study by RNAi showed that, in SK-N-BE(2), whose $\mathrm{N}-\mathrm{Myc}$ expression was in a high level, the N-Myc RNAi and RACK1 RNAi had similar effects on cell migration and proliferation. The repressed expression of $\mathrm{N}-\mathrm{Myc}$ reduced the expression of RACK1, while the repressed expression of RACK1 had no specific effect on expression of N-Myc. These results suggested that $\mathrm{N}-\mathrm{Myc}$ might be an upstream regulator of RACK1 in MYCN gene amplified SK-N-BE(2) cells, which subsequently induced migration and proliferation.

Increasing evidence has been reported on the activation of Akt playing an important role in promoting survival of neuroblastoma $(23,24,29)$. Our results revealed that the activation of Akt was increased by downregulation of N-Myc in MYCN gene amplified neuroblastoma cells, which might be involved in the upregulation of cell proliferation. These anti-apoptosis and pro-proliferation effects of Akt activation might contribute to tumor growth. In our present research, the activations of Akt and ERK1/2 were not changed by the repressed expression of RACK1, which were not consistent with the effect of RACK1 in other tumor cells, such as human colon cells (9) and breast carcinoma (17). The exact molecular mechanism among N-Myc, RACK1 and Akt activation, and their roles in apoptosis, proliferation and migration of neuroblastoma cells still needs further research in the future.

In summary, our data suggested that RACK1 is expressed in the neuroblastoma cells, and positively regulated cell migration and proliferation via modulating the activation of Src on Tyr416 residue. In MYCN gene amplified neuroblastoma cells, RACK1 might be a downstream signaling molecule of $\mathrm{N}-\mathrm{Myc}$ and involved in tumor cell migration and proliferation regulated by N-Myc. Our findings may provide a tool for the evaluation of the invasive potential of neuroblastoma and may provide a new therapeutic target.

\section{Acknowledgements}

This research was supported by grants from the National Natural Science Foundation of China, No. 30801200.

\section{References}

1. Maris JM, Hogarty MD, Bagatell R and Cohn SL: Neuroblastoma. Lancet 369: 2106-2120, 2007.
2. Modak S and Cheung NK: Neuroblastoma: therapeutic strategies for a clinical enigma. Cancer Treat Rev 36: 307-317, 2010.

3. Cotterill SJ, Pearson AD, Pritchard J, Foot AB, Roald B, Kohler JA and Imeson J: Clinical prognostic factors in 1277 patients with neuroblastoma: results of The European Neuroblastoma Study Group 'Survey' 1982-1992. Eur J Cancer 36: 901-908, 2000.

4. Grovas A, Fremgen A, Rauck A, Ruymann FB, Hutchinson CL, Winchester DP and Menck HR: The National Cancer Data Base report on patterns of childhood cancers in the United States. Cancer 80: 2321-2232, 1997.

5. Garaventa A, Parodi S, De Bernardi B, Dau D, Manzitti C, Conte M, Casale F, Viscardi E, Bianchi M, D'Angelo P, Zanazzo GA, Luksch R, Favre C, Tamburini A and Haupt R: Outcome of children with neuroblastoma after progression or relapse. A retrospective study of the Italian neuroblastoma registry. Eur J Cancer 45: 2835-2842, 2009.

6. Ara T and DeClerck YA: Mechanisms of invasion and metastasis in human neuroblastoma. Cancer Metastasis Rev 25: 645-657, 2006.

7. Zaizen Y, Taniguchi S and Suita S: The role of cellular motility in the invasion of human neuroblastoma cells with or without $\mathrm{N}$-myc amplification and expression. J Pediatr Surg 33: 1765-1770, 1998

8. Yoon KJ and Danks MK: Cell adhesion molecules as targets for therapy of neuroblastoma. Cancer Biol Ther 8: 306-311, 2009.

9. Mamidipudi V and Cartwright CA: A novel pro-apoptotic function of RACK1: suppression of Src activity in the intrinsic and Akt pathways. Oncogene 28: 4421-4433, 2009.

10. Dorn GW II and Mochly-Rosen D: Intracellular transport mechanisms of signal transducers. Annu Rev Physiol 64: 407-429, 2002.

11. Sklan EH, Podoly E and Soreq H: RACK1 has the nerve to act: structure meets function in the nervous system. Prog Neurobiol 78: 117-134, 2006.

12. Doan AT and Huttenlocher A: RACK1 regulates Src activity and modulates paxillin dynamics during cell migration. Exp Cell Res 313: 2667-2679, 2007.

13. Padanilam BJ and Hammerman MR: Ischemia-induced receptor for activated C kinase (RACK1) expression in rat kidneys. Am J Physiol 272: F160-F166, 1997.

14. Berns H, Humar R, Hengerer B, Kiefer FN and Battegay EJ: RACK1 is up-regulated in angiogenesis and human carcinomas. FASEB J 14: 2549-2558, 2000.

15. Hermanto U, Zong CS, Li W and Wang LH: RACK1, an insulin-like growth factor I (IGF-I) receptor-interacting protein, modulates IGF-I-dependent integrin signaling and promotes cell spreading and contact with extracellular matrix. Mol Cell Biol 22: 2345-2365, 2002.

16. Buensuceso CS, Woodside D, Huff JL, Plopper GE and O'Toole TE: The WD protein Rack1 mediates protein kinase C and integrin-dependent cell migration. J Cell Sci 114: 1691-1698, 2001.

17. Cao XX, Xu JD, Xu JW, Liu XL, Cheng YY, Wang WJ, Li QQ, Chen Q, Xu ZD and Liu XP: RACK1 promotes breast carcinoma proliferation and invasion/metastasis in vitro and in vivo. Breast Cancer Res Treat 123: 375-386, 2010.

18. Subauste MC, Ventura-Holman T, Du L, Subauste JS, Chan SL, Yu VC and Maher JF: RACK1 downregulates levels of the proapoptotic protein Femlb in apoptosis-resistant colon cancer cells. Cancer Biol Ther 8: 2297-2305, 2009.

19. Cao XX, Xu JD, Liu XL, Xu JW, Wang WJ, Li QQ, Chen Q, Xu ZD and Liu XP: A superior independent predictor for poor clinical outcome in breast cancer. Int J Cancer 127: 1172-1179, 2010.

20. Savini I,D'Angelo I, Annicchiarico-Petruzzelli M,Bellincampi L, Melino G and Avigliano L: Ascorbic acid recycling in N-myc amplified human neuroblastoma cells. Anticancer Res 18: 819-822, 1998 .

21. Burkhart CA, Cheng AJ, Madafiglio J, Kavallaris M, Mili M, Marshall GM, Weiss WA, Khachigian LM, Norris MD and Haber M: Effects of MYCN antisense oligonucleotide administration on tumorigenesis in a murine model of neuroblastoma. J Natl Cancer Inst 95: 1394-1403, 2003.

22. Kang JH, Rychahou PG, Ishola TA, Qiao J, Evers BM and Chung DH: MYCN silencing induces differentiation and apoptosis in human neuroblastoma cells. Biochem Biophys Res Commun 351: 192-197, 2006.

23. Opel D, Poremba C, Simon T, Debatin KM and Fulda S: Activation of Akt predicts poor outcome in neuroblastoma. Cancer Res 67: 735-745, 2007. 
24. Chai H, Luo AZ, Weerasinghe $P$ and Brown RE: Sorafenib downregulates ERK/Akt and STAT3 survival pathways and induces apoptosis in a human neuroblastoma cell line. Int J Clin Exp Pathol 3: 408-415, 2010.

25. Ryu SJ, Cho KA, Oh YS and Park SC: Role of Src-specific phosphorylation site on focal adhesion kinase for senescenceassociated apoptosis resistance. Apoptosis 11: 303-313, 2006.

26. Brunton VG and Frame MC: Src and focal adhesion kinase as therapeutic targets in cancer. Curr Opin Pharmacol 8: 427-432, 2008.

27. Beierle EA, Ma X, Trujillo A, Kurenova EV, Cance WG and Golubovskaya VM: Inhibition of focal adhesion kinase and src increases detachment and apoptosis in human neuroblastoma cell lines. Mol Carcinog 49: 224-234, 2010
28. Hishiki T, Saito T, Sato Y, Mitsunaga T, Terui E, Matsuura G, Saito E, Shibata R, Mise N, Yokoyama Y and Yoshida H: Src kinase family inhibitor PP2 induces aggregation and detachment of neuroblastoma cells and inhibits cell growth in a PI3 kinase/Akt pathway-independent manner. Pediatr Surg Int 27: 225-230, 2011.

29. Li Z, Tan F, Liewehr DJ, Steinberg SM and Thiele CJ: In vitro and in vivo inhibition of neuroblastoma tumor cell growth by AKT inhibitor perifosine. J Natl Cancer Inst 102: 758-770, 2010. 